\title{
OPEN
}

Published online: 22 January 2020

\section{Author Correction: A multigene and morphological analysis expands the diversity of the seabod shrimp Xiphopenaeus Smith, 1869 (Decapoda: Penaeidae), with descriptions of two new species}

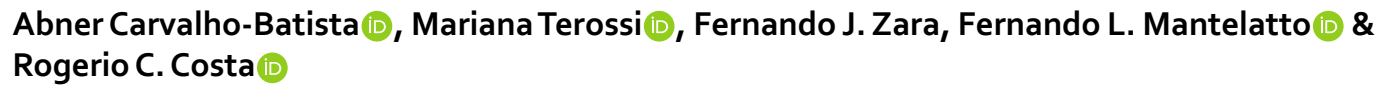

Correction to: Scientific Reports https://doi.org/10.1038/s41598-019-51484-3, published online 25 October 2019

This Article contains multiple typographical errors.

In the Results section, under the subheading 'General description of petasma and appendix masculina of the genus Xiphopenaeus':

"the central region of the ventral face is concave or convex and has rows of spines with varied distribution"

should read:

"the central ventral surface is concave or convex and has rows of spines with varied distribution"

In the legend of Figure 6:

“junction between the endopods with cincinuli (seta); C, Xiphopenaeus kroyeri (CCDB 5019): appendix masculina in dorsal view (DS - dorsal surface; RP - rounded projection); D, Xiphopenaeus kroyeri (CCDB 5019): appendix masculina in ventral view (VS - ventral surface; RP - rounded projection; white arrow indicating the row of spines of the posterior margin).

should read:

"junction between the endopods with cincinuli; C, Xiphopenaeus kroyeri (CCDB 5019): appendix masculina in dorsal view (DS - dorsal surface; RP - rounded projection); D, Xiphopenaeus kroyeri (CCDB 5019): appendix masculina in ventral view (VS - ventral surface; RP - rounded projection; white arrow indicating the central convex surface).

In the Results section, under the subheading 'General description of petasma and appendix masculina of the genus Xiphopenaeus':

"The posterior margin of the ventral surface is covered by small sparse spines, with a few rows in the central convex region"

should read: 
"The posterior margin of the ventral surface is covered by small sparse spines, with a few rows in the central convex surface"

In the legend of Figure 8:

"Appendix masculina in ventral view; the black arrow indicates the row of spines of the posterior margin, white arrows indicate the convex central region; (H) Appendix masculina in ventral view; the black arrow indicates the row of spines of the posterior margin, white arrows indicate the convex central region"

should read:

"Appendix masculina in ventral view; the black arrow indicates the row of spines of the posterior margin, white arrows indicate the convex central surface; $(\mathrm{H})$ Appendix masculina in ventral view; the black arrow indicates the row of spines of the posterior margin, white arrows indicate the convex central surface. (AM - appendix masculina; CxVS - central convex ventral surface; D - distal region of the distolateral projection; DLP - distolateral projection; E - endopod; P - proximal region of the distolateral projection; RP - round projection of appendix masculina)."

In the legend of Figures 11, 13 and 14:

"Appendix masculina in ventral view; the black arrow indicates the row of spines of the posterior margin, white arrows indicate the convex central region; $(\mathrm{H})$ Appendix masculina in ventral view; the black arrow indicates the row of spines of the posterior margin, white arrows indicate the convex central region"

should read:

"Appendix masculina in ventral view; the black arrow indicates the row of spines of the posterior margin, white arrows indicate the convex central surface; $(\mathrm{H})$ Appendix masculina in ventral view; the black arrow indicates the row of spines of the posterior margin, white arrows indicate the convex central surface. (AM - appendix masculina; CcVS - central concave ventral surface; D - distal region of the distolateral projection; DLP - distolateral projection; $\mathrm{E}$ - endopod; P - proximal region of the distolateral projection; RP - round projection of appendix masculina)."

In the legend of Figure 15:

“6-Colombia ()"

should read:

“6-Colombia $\left(10^{\circ} 52^{\prime} 30^{\prime \prime} \mathrm{N} ; 74^{\circ} 24^{\prime} 10.800^{\prime \prime} \mathrm{W}\right) ”$

In the results section, in the Xiphopenaeus dincao nov. sp. section under the subheading 'Description':

"The posterior margin of the ventral surface is covered by small sparse spines, with a few rows in the central convex region"

should read:

"The posterior margin of the ventral surface is covered by small sparse spines, with a few rows in the central concave surface"

In the Methods section, under the subheading 'Morphological Assessment':

“(Catalog numbers - 5019, 5247)”

should read:

“(5019, 5247, 5461, 6499)”

Open Access This article is licensed under a Creative Commons Attribution 4.0 International License, which permits use, sharing, adaptation, distribution and reproduction in any medium or format, as long as you give appropriate credit to the original author(s) and the source, provide a link to the Creative Commons license, and indicate if changes were made. The images or other third party material in this article are included in the article's Creative Commons license, unless indicated otherwise in a credit line to the material. If material is not included in the article's Creative Commons license and your intended use is not permitted by statutory regulation or exceeds the permitted use, you will need to obtain permission directly from the copyright holder. To view a copy of this license, visit http://creativecommons.org/licenses/by/4.0/.

(c) The Author(s) 2020 